\title{
Evaluation of in vitro and in vivo therapeutic antitumor efficacy of transduction of polo-like kinase 1 and heat shock transcription factor 1 small interfering RNA
}

\author{
YOSHIYUKI HATTORI $^{1}$, TAKUTO KIKUCHI ${ }^{1}$, KEI-ICHI OZAKI ${ }^{2}$ and HIRAKU ONISHI ${ }^{1}$ \\ ${ }^{1}$ Department of Drug Delivery Research, Hoshi University, Tokyo 142-8501; ${ }^{2}$ Education and Research Center for \\ Fundamental Pharmaceutical Sciences, Osaka University of Pharmaceutical Sciences, Osaka 569-1094, Japan
}

Received April 6, 2017; Accepted July 27, 2017

DOI: $10.3892 /$ etm.2017.5060

\begin{abstract}
Mitotic progression is regulated by the phosphorylation of heat shock transcription factor 1 (HSF1) by polo-like kinase 1 (PLK1); however, this interaction is often deregulated in tumors. High expression levels of PLK1 and HSF1 have been observed in various types of human cancer. In the present study, it was investigated whether small interfering (si)RNA against PLK1 or HSF1 could suppress tumor growth in vitro and in vivo. In vitro transfection of PLK1 and HSF1 siRNA into PKL1- and HSF1-positive human breast tumor MDA-MB-231 and human cervical carcinoma HeLa cells inhibited cell growth via suppression of PLK1 and HSF1 mRNA expression, respectively. However, the transfection of PLK1 or HSF1 siRNA did not significantly affect the cytotoxicity of doxorubicin in HeLa cells. Furthermore, injection of PKL1 or HSF1 siRNA into mice with liver HeLa metastasis suppressed tumor growth. From these findings, PLK1 and HSF1 may be considered to be promising targets for antitumor therapy.
\end{abstract}

\section{Introduction}

Heat shock factor 1 (HSF1) is a transcription factor that is strongly conserved from yeast to humans (1). HSF1 has a key role in the cellular response leading to the expression of heat shock proteins (Hsps), which serve to protect cells from damage as a result of cellular insults, including heat and oxidative stress $(1,2)$. However, the heat shock response often becomes deregulated in tumors. HSF1 is expressed at a high level and has a role in carcinogenesis (3). The expression levels of Hsp90, Hsp70 and Hsp27, which are HSF1-target

Correspondence to: Dr Yoshiyuki Hattori, Department of Drug Delivery Research, Hoshi University, 2-4-41 Ebara, Tokyo 142-8501, Japan

E-mail: yhattori@hoshi.ac.jp

Key words: polo-like kinase 1, heat shock transcription factor 1, small interfering RNA, tumor, liver metastasis genes, are increased in mammary tumors, leading to a decrease in cellular apoptosis (4). Polo-like kinase 1 (PLK1) is a serine/threonine protein kinase that acts as an important cell signaling regulator (5). The phosphorylation of HSF1 by PLK1 is a vital step for HSF1 nuclear translocation in response to heat stress (6). PLK1 expression is also elevated in various types of human tumors (7-9). It has been reported that depletion of PLK1 in oral squamous cell carcinoma cells significantly inhibited the expression of HSF1 as well as Hsp70 and Hsp90, and knockdown of PLK1 and HSF1 strongly inhibited cell proliferation (10). Furthermore, transduction of PLK1 small interfering (si)RNA or HSF1 short hairpin (sh)RNA into tumor cells reduced the migration and invasive abilities of the cells $(11,12)$. Therefore, PLK1 and HSF1 may be potential targets in cancer therapy.

Cationic liposomes have often been used for in vivo siRNA delivery into tumors (13). However, following systemic injection of siRNA/cationic liposome complexes (siRNA lipoplexes), electrostatic interactions between positively charged lipoplexes and negatively charged erythrocytes cause agglutination in the blood, and the agglutinates contribute to high levels of entrapment of lipoplexes in the highly extended lung capillaries (14). Recently, we demonstrated that intravenous sequential injection of chondroitin sulfate plus siRNA lipoplexes into mice with liver metastasis could deliver siRNA efficiently to the metastasis without accumulation in the lungs, and suppress the expression of a target gene in the tumor cells $(15,16)$. Therefore, the present study evaluated the therapeutic efficacy of treatment with PLK1 or HSF1 siRNA using a sequential injection method against liver metastasis.

\section{Materials and methods}

Reagents. 1,2-Dioleoyl-3-trimethylammonium-propane (DOTAP) methyl sulfate salt was obtained from Avanti Polar Lipids Inc., (Alabaster, AL, USA). Cholesterol (Chol) and chondroitin sulfate $\mathrm{C}$ sodium salt were purchased from Wako Pure Chemical Industries, Ltd. (Osaka, Japan). All other chemicals were of the finest grade available.

siRNA. Human PLK1 siRNA, human HSF1 siRNA, non-silencing siRNA (Cont siRNA), and cyanine 5.5 
(Cy5.5)-labeled luciferase siRNA (Cy5.5-siRNA) were synthesized by Sigma Genosys (Tokyo, Japan). The siRNA sequences for human PLK1 siRNA were as follows: Sense strand 5'-CCU UGAUGAAGAAGAUCACTT-3' and antisense strand 5'-GUG AUCUUCUUCAUCAAGGTT-3' (17). The siRNA sequences of the human HSF1 siRNA were: Sense strand 5'-GAACGA CAGUGGCUCAGCAUU-3' and antisense strand 5'-UGC UGAGCCACUGUCGUUCUU-3' (17). The siRNA sequences of the Cont siRNA as a negative control for PLK1 siRNA or HSF1 siRNA were: Sense strand 5'-GUACCGCACGUCAUU CGUAUC-3' and antisense strand 5'-UACGAAUGACGUGCG GUACGU-3'. The siRNA sequences of the luciferase siRNA were: Sense strand 5'-GUGGAUUUCGAGUCGUCUUAA-3' and antisense strand 5'-AAGACGACUCGAAAUCCACAU-3. In Cy5.5-siRNA, Cy5.5 dye was conjugated at the 5'-end of the sense strand.

Cell culture. Human breast cancer MDA-MB-231/Luc cells stably expressing firefly luciferase were obtained from Cell BioLabs, Inc., (San Diego, CA, USA). Tamoxifen-resistant human breast cancer MCF-7-Luc (TamR-Luc\#1) cells stably expressing firefly pGL3 luciferase were donated by Dr Kazuhiro Ikeda (Division of Gene Regulation and Signal Transduction, Research Center for Genomic Medicine, Saitama Medical University, Saitama, Japan). Human cervical carcinoma HeLa-Luc cells stably expressing firefly pGL3 luciferase were obtained from Caliper Life Sciences Co., (Hopkinton, MA, USA).

MCF-7-Luc cells were cultured in Dulbecco's modified Eagle medium (DMEM; Wako Pure Chemical Industries, Ltd.) supplemented with $10 \%$ fetal bovine serum (FBS; Gibco; Thermo Fisher Scientific, Inc., Waltham, MA, USA), $100 \mu \mathrm{g} / \mathrm{ml}$ kanamycin and $0.5 \mathrm{mg} / \mathrm{ml} \mathrm{G} 418$ at $37^{\circ} \mathrm{C}$ in a $5 \% \mathrm{CO}_{2}$ humidified atmosphere. MDA-MB-231-Luc cells were cultured in DMEM supplemented with $10 \%$ FBS and $100 \mu \mathrm{g} / \mathrm{ml} \mathrm{kana-}$ mycin at $37^{\circ} \mathrm{C}$ in a $5 \% \mathrm{CO}_{2}$ humidified atmosphere. HeLa cells were cultured in Eagle's minimum essential medium (Wako Pure Chemical Industries, Ltd.) supplemented with 10\% FBS and $100 \mu \mathrm{g} / \mathrm{ml}$ kanamycin at $37^{\circ} \mathrm{C}$ in a $5 \% \mathrm{CO}_{2}$ humidified atmosphere.

Measurement of PLK1 and HSF1 expression level in vitro. For investigation of PLK1 and HSF1 mRNA expression levels in tumorcells, total RNA was isolated from MDA-MB-231, MCF-7 and HeLa cells using NucleoSpin RNA (Macherey-Nagel, GmbH, Düren, Germany). First strand cDNA was synthesized from $2 \mu \mathrm{g}$ of total RNA using PrimeScript RTase (Takara Bio, Inc., Otsu, Japan). For polymerase chain reaction (PCR), the $20-\mu 1$ reaction volume contained: $1 \mu 1$ synthesized cDNA, 10 pmol of each specific primer pair and $0.25 \mathrm{U}$ Ex Taq DNA polymerase (Takara Bio, Inc.), with PCR buffer containing $1.5 \mathrm{mM} \mathrm{MgCl}{ }_{2}$ and $0.25 \mathrm{mM}$ of each dNTP. The profile of PCR amplification for PLK1 and GAPDH cDNA consisted of denaturation at $95^{\circ} \mathrm{C}$ for $0.5 \mathrm{~min}$, primer annealing at $58^{\circ} \mathrm{C}$ for $0.5 \mathrm{~min}$ and elongation at $72^{\circ} \mathrm{C}$ for $1 \mathrm{~min}$, for 30 cycles. For HSF1, the thermocycling conditions consisted of denaturation at $95^{\circ} \mathrm{C}$ for $0.5 \mathrm{~min}$, primer annealing at $63^{\circ} \mathrm{C}$ for $0.5 \mathrm{~min}$ and elongation at $72^{\circ} \mathrm{C}$ for $1 \mathrm{~min}$, for 30 cycles. Human PLK1 cDNA (154 bp) was amplified using the following primers: Human PLK1-FW 5'-CTCAACACGCCTCATCCTC-3' and human PLK1-RW 5'-GTGCTCGCTCATGTAATTGC-3' (18). Human HSF1 cDNA (190 bp) was amplified using the primers: Human HSF1-FW, (5'-CCGGCGGGAGCATAGACGAGA GG-3') and human HSF1-RW (5'-GACGGAGGCGGGGGC AGGTTCACT-3') (19). Human GAPDH cDNA (820 bp) was amplified using the primers: Human GAPDH-FW (5'-ATG ACCCCTTCATTGACCTC-3') and human GAPDH-RW (5'-AAGTGGTCGTTGAGGGCAAT-3'). Their PCR products were analyzed by $18 \%$ acrylamide gel electrophoresis in Tris-borate-EDTA buffer (Wako Pure Chemical Industries, Ltd.), and were visualized by ethidium bromide staining.

Transfection with siRNA. For knockdown of PLK1 or HSF1 mRNA by transfection with PLK1 siRNA or HSF1 siRNA, MDA-MB-231 and HeLa cells were plated into 6-well culture dishes at a density of $3 \times 10^{5}$ cells/well. The cells were transfected with $50 \mathrm{nM}$ Cont, PLK1 or HSF1 siRNA using Lipofectamine RNAiMax reagent (Invitrogen; Thermo Fisher Scientific, Inc.). A total of $24 \mathrm{~h}$ after transfection, total RNA was isolated using NucleoSpin RNA and then first strand cDNA was synthesized from $2 \mu \mathrm{g}$ of total RNA using PrimeScript RTase. Quantitative (q)PCR was performed using a Roche Light Cycler 96 system (Roche Diagnostics, Basel, Switzerland) and TaqMan Gene expression assays (PLK1, Hs00983227_m1; HSF1, Hs00232134_m1; GAPDH, Hs02786624_g1; all Applied Biosystems; Thermo Fisher Scientific, Inc.). The thermocycling conditions consisted of an initial denaturation at $95^{\circ} \mathrm{C}$ for $600 \mathrm{sec}$, and 45 cycles of denaturation at $95^{\circ} \mathrm{C}$ for $10 \mathrm{sec}$, and primer annealing and extension at $60^{\circ} \mathrm{C}$ for $30 \mathrm{sec}$ (two step amplification). Samples were run in triplicate, and the expression levels of PLK1 and HSF1 mRNA were normalized by the amount of GAPDH mRNA in the same sample, and analyzed using the $2^{-\Delta \Delta C q}$ method (20).

Cell growth inhibition. MCF-7, MDA-MB-231 and HeLa cells were seeded in 96 -well plates at a density of $2 \times 10^{4}$ cells per well $24 \mathrm{~h}$ prior to transfection. Cells at confluences of $50 \%$ in the well were transfected with 2.5, 5, 10, 20, 30, 40 and $50 \mathrm{nM}$ Cont siRNA, PLK1 siRNA or HSF1 siRNA using Lipofectamine RNAiMax reagent and then incubated for $48 \mathrm{~h}$ at $37^{\circ} \mathrm{C}$. In combined treatment with doxorubicin (DXR; LC Laboratories, Woburn, MA, USA), the cells were incubated for $24 \mathrm{~h}$ at $37^{\circ} \mathrm{C}$ after transfection at $50 \mathrm{nM}$ Cont siRNA, PLK1 siRNA or HSF1 siRNA using Lipofectamine RNAiMax reagent, and then treated with various concentrations (0.016-0.5 $\mu \mathrm{M})$ of DXR as reported previously (21). A total of $6 \mathrm{~h}$ after incubation, the medium containing free DXR was changed for fresh medium without DXR, and then incubated for another $18 \mathrm{~h}$ at $37^{\circ} \mathrm{C}$. The cell number was determined using a Cell Counting kit-8 (Dojindo Molecular Technologies, Inc., Kumamoto, Japan). Cell viability was expressed relative to the absorbance of untreated cells at $450 \mathrm{~nm}$.

Preparation of liposomes and lipoplexes. Cationic liposomes were prepared from DOTAP/Chol at a molar ratio of 1:1 using a thin-film hydration method, as reported previously (22). To prepare siRNA/cationic liposome complexes (siRNA lipoplexes), the cationic liposome suspension was mixed with siRNA by vortexing for $10 \mathrm{sec}$ at a charge ratio (+:-) of 4:1, and left for $15 \mathrm{~min}$ at room temperature. The theoretical charge 
ratio (+:-) of cationic liposome to siRNA was calculated as the molar ratio of DOTAP nitrogen to siRNA phosphate.

The particle size distributions of liposomes and lipoplexes were measured using a light-scattering photometer (ELS-Z2; Otsuka Electronics Co., Ltd., Osaka, Japan) at $25^{\circ} \mathrm{C}$ after diluting the dispersion to an appropriate volume with water. The $\zeta$-potentials were measured using ELS-Z2 at $25^{\circ} \mathrm{C}$ after diluting the dispersion with an appropriate volume of water. Cationic liposomes were $\sim 114 \mathrm{~nm}$ in size and had a $\zeta$-potential of $\sim 52 \mathrm{mV}$. The lipoplex size was $\sim 312 \mathrm{~nm}$ and the $\zeta$-potential was $\sim 40 \mathrm{mV}$.

Liver metastasis model. All animal experiments were performed with approval from the Institutional Animal Care and Use Committee of Hoshi University (Tokyo, Japan). A total of 9 mice were housed in a temperature- $\left(24^{\circ} \mathrm{C}\right)$ and humidity- $(55 \%)$ controlled room with a $12 \mathrm{~h}$ light/dark cycle (lights on at 8:00 a.m.) with ad libitum access to food and water. To generate mice with liver metastasis, $1.0 \times 10^{6} \mathrm{HeLa}$ cells suspended in $50 \mu \mathrm{lPBS}$ ( $\mathrm{pH} 7.4$ ) containing 50\% reconstituted basement membrane (Matrigel; BD Biosciences, Franklin Lakes, NJ, USA) were inoculated into the spleen of female SCID-Beige mice (18-20 g; 8 weeks old; CB17.B6-Prkdc ${ }^{\text {scid }}$ Lyst $^{\text {bg-J }} / \mathrm{Crl}$; Oriental Yeast Co. Ltd., Tokyo, Japan).

Biodistribution of siRNA following intravenous injection of siRNA lipoplexes into mice. For efficient delivery of siRNA into liver metastasis, lipoplexes with $50 \mu \mathrm{g}$ Cy5.5-siRNA were administered intravenously into mice with HeLa-liver metastasis at $1 \mathrm{~min}$ after the intravenous injection of $1 \mathrm{mg}$ chondroitin sulfate (Wako Pure Chemical Industries, Ltd.) at 10 days after inoculation of HeLa-Luc cells, as reported previously (sequential injection method) (15,16). A total of $1 \mathrm{~h}$ after injection, the mice were sacrificed, and Cy5.5 fluorescent imaging of the tissues was performed using a NightOWL LB981 NC100 system (Berthold Technologies, Bad Wildbad, Germany). In Cy5.5 fluorescent imaging, the excitation and emission filters were set at 630/20 and $680 / 30 \mathrm{~nm}$, respectively. The exposure time for fluorescence was $5 \mathrm{sec}$. A grayscale body-surface reference image was collected using a NightOWL LB981 CCD camera (Berthold Technologies). The images were analyzed using IndiGo2 software (version 2.0.1.0; Berthold Technologies) provided with the in vivo imaging system.

In vivo therapy for liver HeLa metastasis. On days 8, 10, 12 and 14 after inoculation of HeLa cells into the spleens of mice, $50 \mu \mathrm{g}$ of Cont siRNA, PLK1 siRNA or HSF1 siRNA were administered to mice using a sequential injection method as described above $(15,16)$. On day 16 after inoculation, mice were sacrificed by cervical dislocation, and then the excised livers and spleens were weighed.

Statistical analysis. The statistical significance of differences between mean values was determined using Student's t-test using GraphPad Prism (version 4.0; GraphPad Software, Inc., La Jolla, CA, USA). Multiple measurement comparisons were performed by one-way analysis of variance on ranks with post hoc Tukey-Kramer's test. $\mathrm{P}<0.05$ was considered to indicate a statistically significant difference.

\section{Results and Discussion}

Expression level of HSF1 and PLK1 mRNA. For investigation of HSF1 and PLK1 mRNA expression levels in cultured cells, three human tumor cell lines, (MDA-MB-231, MCF-7 and HeLa cells) were used. As demonstrated in Fig. 1A, PLK1 and HSF1 mRNA were expressed strongly in MDA-MB-231 and HeLa cells, but weakly or not at all in MCF-7 cells. Therefore, in subsequent experiments, MDA-MB-231 and HeLa cells were used as the cell lines that expressed HSF1 and PLK1 mRNA at high levels.

Subsequently, whether the expression level of PLK1 and HSF1 mRNA was decreased by transfecting PLK1 siRNA and HSF1 siRNA into the cells, respectively, was investigated. Lipofectamine RNAiMax was used as an in vitro transfection reagent for siRNA. When transfected into MDA-MB-231 and HeLa cells, PLK1 siRNA and HSF1 siRNA significantly inhibited the expression of PLK1 and HSF1 mRNA $(\mathrm{P}<0.01)$, respectively, compared with the levels in the untreated or Cont siRNA groups (Fig. 1B and C). In contrast, transfection of Cont siRNA or HSF1 siRNA did not significantly affect the expression of PLK1 mRNA in MDA-MB-231 and HeLa cells (Fig. 1B), and transfection of Cont siRNA or PLK1 siRNA did not significantly affect HSF1 mRNA compared with the levels in the untreated cells (Fig. 1C). These results indicated that PLK1 siRNA and HSF1 siRNA could specifically suppress the expression of PLK1 and HSF1 mRNA in the cells, respectively, and suppression of PLK1 mRNA did not affect the expression level of HSF1 mRNA in the cells.

Effect of HSF1 siRNA and PLK1 siRNA on cell viability. To examine whether transfection of HSF1 siRNA or PLK1 siRNA into tumor cells could inhibit tumor growth, cell viability was measured $48 \mathrm{~h}$ after transfection of HSF1 siRNA or PLK1 siRNA into MDA-MB-231, HeLa and MCF-7 cells. In MCF-7 cells, transfection with HSF1 siRNA did not significantly affect cell viability compared with transfection with Cont siRNA. However, transfection with PLK1 siRNA significantly suppressed cell viability at concentrations of 5, 10, 20, 30, 40 and $50 \mathrm{nM}$ siRNA compared with the viability of cells transfected with Cont siRNA (Fig. 2). These results suggested that HSF1 siRNA may not be effective for growth inhibition of MCF-7 cells. In contrast, MDA-MB-231 and HeLa cells transfected with HSF1 siRNA demonstrated a significant decrease in viability at some concentrations compared with those transfected with Cont siRNA ( $\mathrm{P}<0.05$; Figs. 3 and 4). Transfection with PLK1 siRNA significantly inhibited cell growth at all concentrations in MDA-MB-231 and HeLa cells compared with those transfected with Cont siRNA ( $\mathrm{P}<0.01$; Figs. 3 and 4), indicating that suppression of PLK1 expression strongly affected in vitro growth in tumor cells that expressed PLK1 mRNA at high levels.

Following this, it was evaluated whether transfection with PLK1 siRNA or HSF1 siRNA could increase the growth inhibitory effect of DXR treatment in HeLa cells. The combined treatment of HSF1 siRNA with DXR exhibited additive cytotoxicity in HeLa cells compared with HSF1 siRNA treatment alone (Fig. 5). However, the combined treatment of PLK1 siRNA with DXR did not exhibit a significant synergistic or additive cytotoxicity in HeLa cells compared with PLK1 siRNA treatment 
A

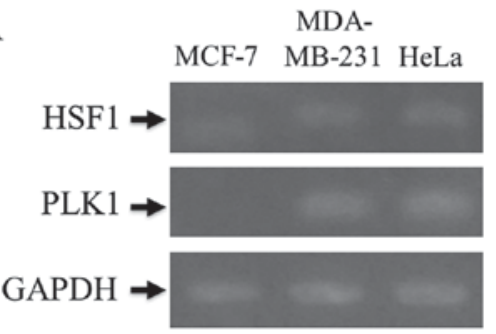

B

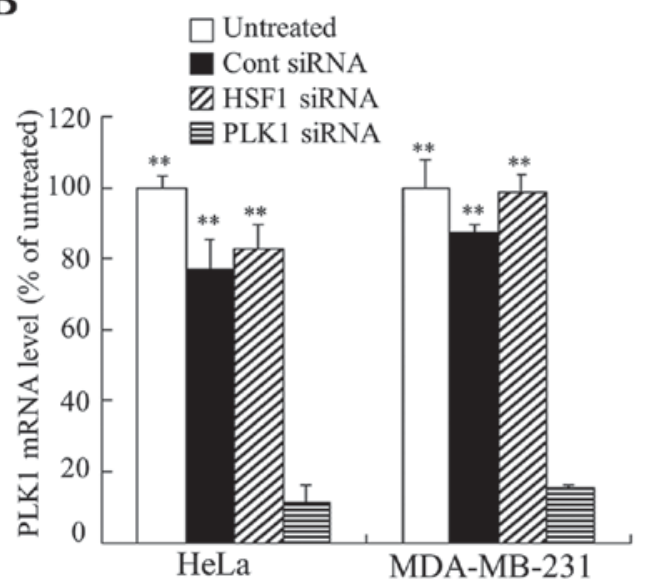

$\mathrm{C}$

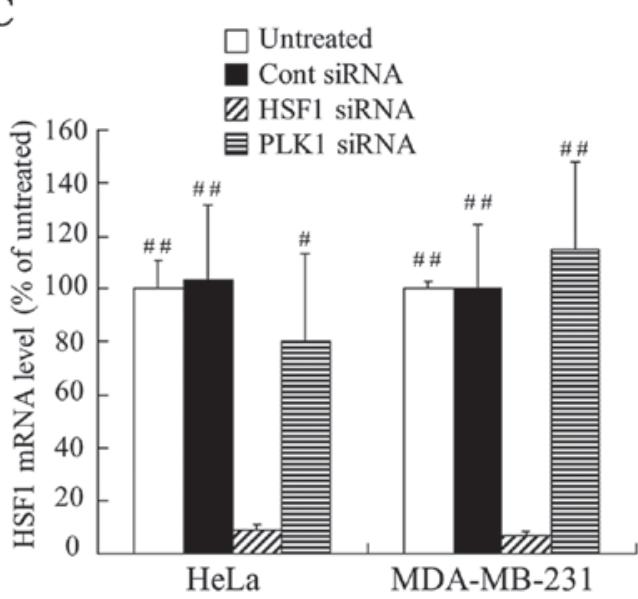

Figure 1. Expression of PLK1 and HSF1 mRNA in MCF-7, MDA-MB-231 and HeLa cells, and suppression of PLK1 or HSF1 mRNA expression by transfection with siRNA in MDA-MB-231 and HeLa cells. (A) The expression levels of HSF1 and PLK1 mRNA in MCF-7, MDA-MB-231 and HeLa cells were analyzed by RT-PCR. MDA-MB-231 and HeLa cells were transfected with $50 \mathrm{nM}$ Cont, HSF1 or PLK1 siRNA using Lipofectamine RNAiMax reagent, and the expression levels of (B) PLK1 and (C) HSF1 mRNA in the cells were analyzed by RT-quantitative PCR. Data are presented as the mean + standard deviation $(\mathrm{n}=3) .{ }^{* *} \mathrm{P}<0.01$ vs. PLK1 siRNA; ${ }^{*} \mathrm{P}<0.05$ and ${ }^{\# \#} \mathrm{P}<0.01$ vs. HSF1 siRNA. PLK1, polo-like kinase 1; HSF1, heat shock transcription factor 1; RT-PCR, reverse transcription polymerase chain reaction; siRNA, small interfering RNA; Cont, control.

A

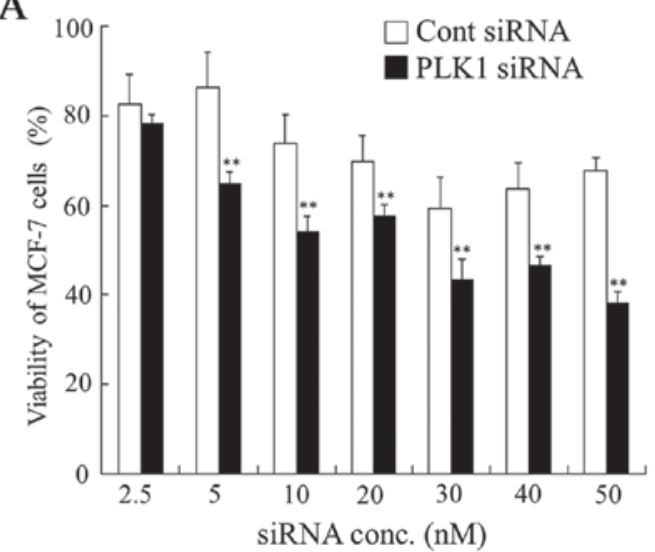

B

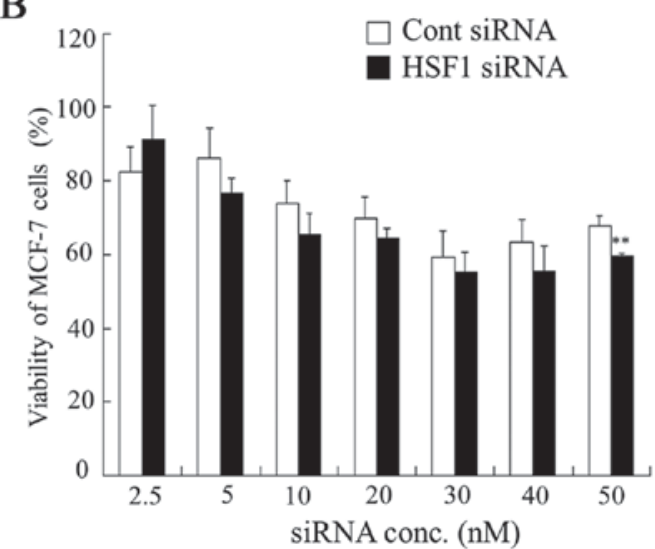

Figure 2. Dose dependence of anti-proliferative activities $48 \mathrm{~h}$ after transfection with PLK1 or HSF1 siRNA into MCF-7 cells. At $48 \mathrm{~h}$ after transfection with (A) PLK1 or (B) HSF1 siRNA at 2.5, 5, 10, 20, 30, 40 and $50 \mathrm{nM}$, cell viability was measured. Data are presented as the mean + standard deviation (n=4). ${ }^{* *} \mathrm{P}<0.01$ vs. Cont siRNA. PLK1, polo-like kinase 1; HSF1, heat shock transcription factor 1; siRNA, small interfering RNA; Cont, control; conc, concentration.

alone (Fig. 5). These data indicated that a reduction in PLK1 or HSF1 expression did not increase the sensitivity to DXR in HeLa cells. Therefore, for the in vivo experiment for evaluation of therapeutic efficacy for tumor metastasis, we decided to inject PKL1 siRNA or HSF1 siRNA without DXR.

Biodistribution of siRNA following injections of siRNA lipoplexes. Recently, we demonstrated that intravenous sequential injection of chondroitin sulfate plus siRNA lipoplexes into mice with liver metastasis could deliver siRNA efficiently to the metastasis without accumulation in the lungs, and suppress the expression of a target gene in the tumor cells $(15,16)$. Therefore, the present study evaluated the therapeutic efficacy of treatment with PLK1 siRNA or HSF1 siRNA using the sequential injection method against HeLa-liver metastasis. First, the biodistribution of siRNA following sequential injections of chondroitin sulfate plus siRNA lipoplexes into mice with HeLa-liver metastasis was investigated. To generate 
A

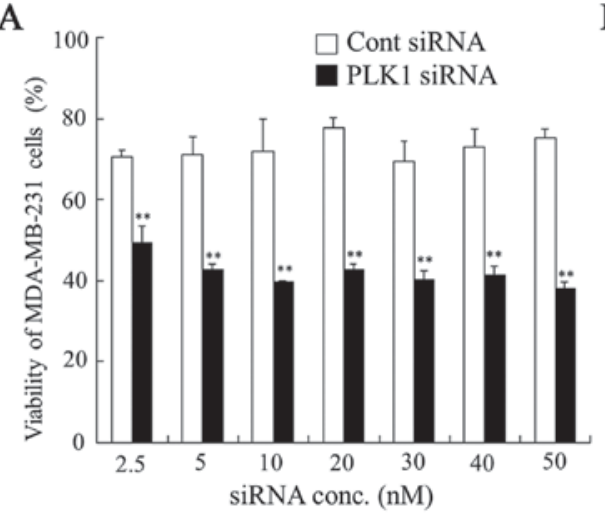

B

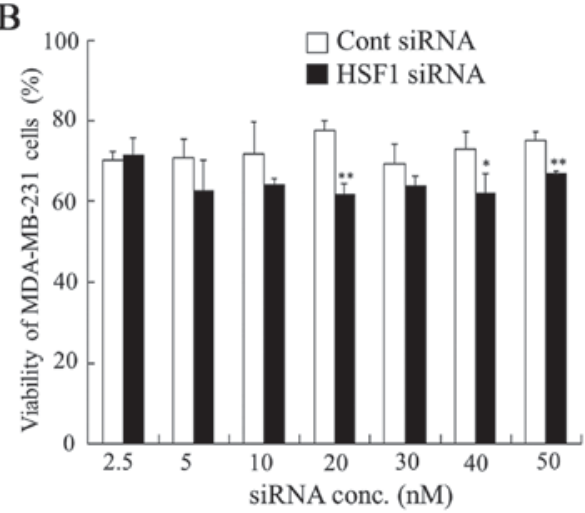

Figure 3. Dose dependence of anti-proliferative activities $48 \mathrm{~h}$ after transfection with PLK1 or HSF1 siRNA into MDA-MB-231 cells. At $48 \mathrm{~h}$ after transfection with (A) PLK1 or (B) HSF1 siRNA at 2.5, 5, 10, 20,30, 40 and $50 \mathrm{nM}$, cell viability was measured. Data are presented as the mean + standard deviation (n=4). ${ }^{*} \mathrm{P}<0.05$ and ${ }^{* *} \mathrm{P}<0.01$ vs. Cont siRNA. PLK1, polo-like kinase 1; HSF1, heat shock transcription factor 1; siRNA, small interfering RNA; Cont, control; conc, concentration.
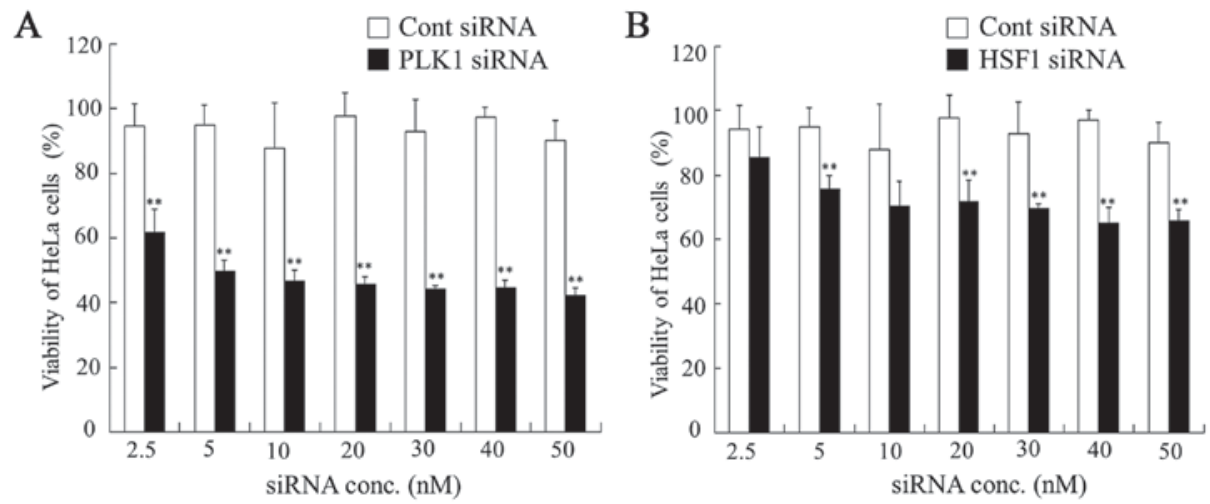

Figure 4. Dose dependence of anti-proliferative activities $48 \mathrm{~h}$ after transfection with PLK1 or HSF1 siRNA into HeLa cells. At $48 \mathrm{~h}$ after transfection with (A) PLK1 or (B) HSF1 siRNA at 2.5, 5, 10, 20,30, 40 and $50 \mathrm{nM}$, cell viability was measured. Data are presented as the mean + standard deviation (n=4). ${ }^{* *} \mathrm{P}<0.01$ vs. Cont siRNA. PLK1, polo-like kinase 1; HSF1, heat shock transcription factor 1; siRNA, small interfering RNA; Cont, control; conc, concentration.

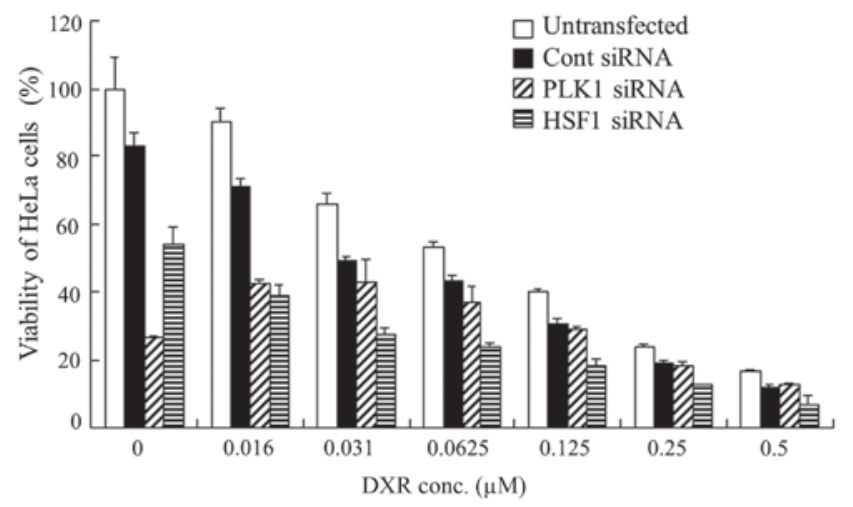

Figure 5. Dose effect of DXR on cytotoxicity in HeLa cells at $24 \mathrm{~h}$ after transfection with PLK1 or HSF1 siRNA. The cells were transfected with $50 \mathrm{nM}$ Cont siRNA, PLK1 siRNA or HSF1 siRNA for $24 \mathrm{~h}$ and then treated with various concentrations of DXR for an additional $6 \mathrm{~h}$. The medium containing DXR was changed for fresh medium without DXR, and then incubated for another $18 \mathrm{~h}$. The number of viable cells was determined using a Cell Counting kit- 8 assay. Data are presented as the mean + standard deviation $(n=4)$. DXR, doxorubicin; PLK1, polo-like kinase 1; HSF1, heat shock transcription factor 1; siRNA, small interfering RNA; Cont, control; conc, concentration.

mice with liver metastasis, HeLa cells were inoculated into the spleens of mice. As demonstrated in Fig. 6A, siRNA was largely accumulated in HeLa-metastasized liver. These results indicated that sequential injections could deliver siRNA effectively into HeLa-metastasized livers of mice.

Therapeutic efficacy against liver HeLa-metastasized tumors. Finally, the present study evaluated the in vivo efficacy of PLK1 siRNA or HSF1 siRNA in inhibiting the growth of liver HeLa-metastasized tumors. Injection of PLK1 siRNA or HSF1 siRNA was performed a total of four times, with 2 days between each injection. The antitumor effect on metastasis was evaluated by measurement of liver weight (mg) (Fig. 6B and C). Injections of PLK1 siRNA suppressed the increase in weight of HeLa-metastasized liver $(1,677 \pm 342 \mathrm{mg})$ compared with those injected with Cont siRNA $(2,567 \pm 453 \mathrm{mg})$; however, this difference was not significant. In contrast, injections of HSF1 siRNA significantly inhibited the increase in weight of metastasized livers $(1,217 \pm 280 \mathrm{mg})$ compared with those injected with Cont siRNA $(\mathrm{P}<0.05)$. Regarding the weight of HeLa-inoculated spleens, significant differences in the weight of spleens were not observed among the different groups (Fig. 6B and C). These results suggested that injection of HSF1 siRNA could inhibit tumor metastasis to the liver and/or tumor growth in liver metastasis. However, it was not clear why HSF1 siRNA did not exhibit strong growth inhibition 
A

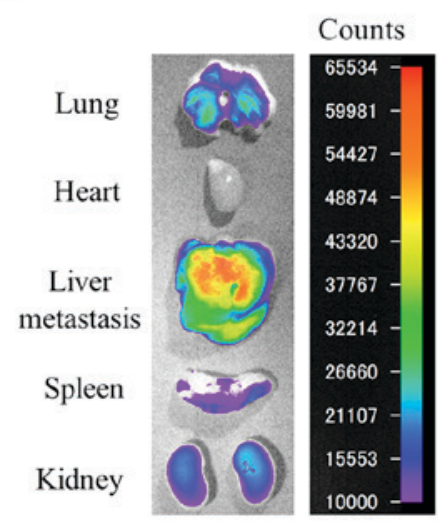

B

$$
\text { HeLa metastasized liver }
$$

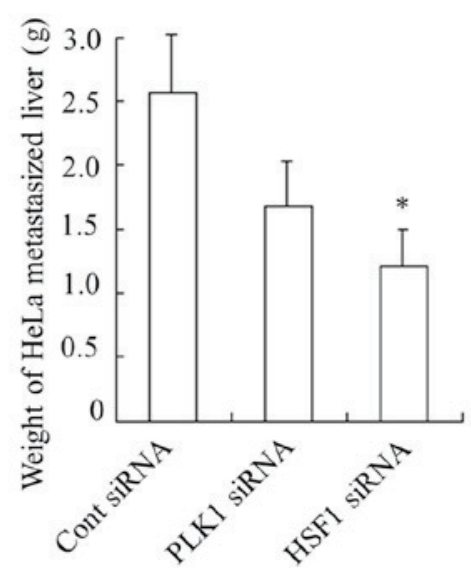

HeLa inoculated spleen

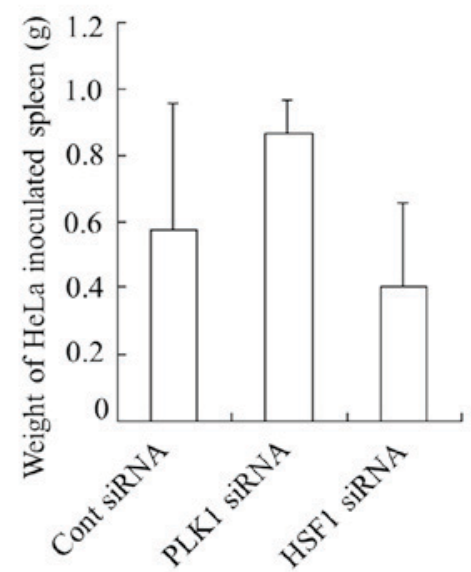

\section{C}

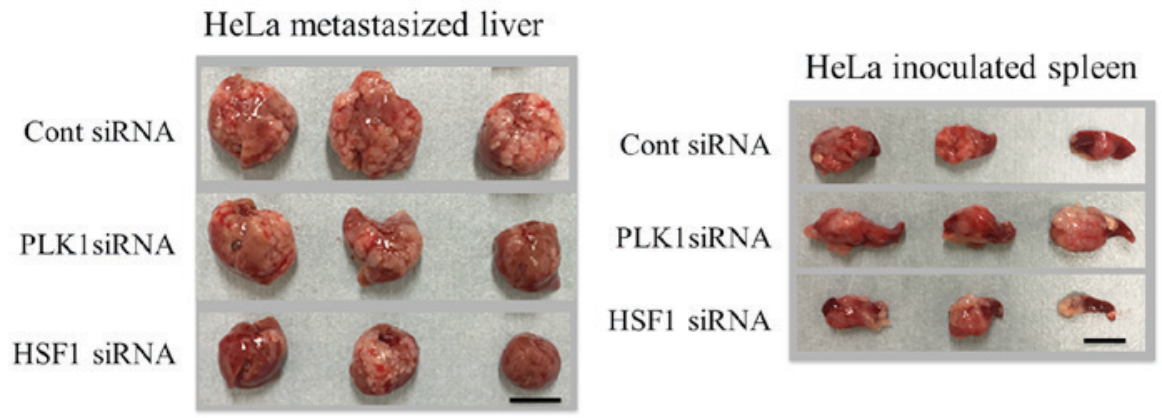

Figure 6. In vivo therapy with PLK1 or HSF1 siRNA for mice with liver HeLa metastasis. (A) Ex vivo images of dissected tissues at $1 \mathrm{~h}$ after sequential injections of chondroitin sulfate plus siRNA lipoplexes into mice with liver HeLa metastasis. Lipoplexes with $50 \mu \mathrm{g}$ of Cy5.5-siRNA were administered intravenously into mice at $1 \mathrm{~min}$ after intravenous injection of $1 \mathrm{mg}$ chondroitin sulfate. The exposure time for the detection of Cy5.5 fluorescence was 5 sec. Fluorescence intensity is illustrated using a color-coded scale (red is maximum, purple is minimum). (B and C) Cont, HSF1 or PLK1 siRNA was administered by sequential injection of chondroitin sulfate plus siRNA lipoplexes on days 8, 10, 12 and 14 after inoculation. (B) Mice were sacrificed at day 16, and then the excised livers and spleens were weighed. (C) Gross appearances of excised livers and spleens at day 16 . Scale bar=1 cm. Data are presented as the mean + standard deviation $(\mathrm{n}=3)$. ${ }^{*} \mathrm{P}<0.05$ vs. Cont siRNA. PLK1, polo-like kinase 1; HSF1, heat shock transcription factor 1; siRNA, small interfering RNA; Cont, control.

in vitro compared with PLK1 siRNA (Fig. 4). HSF1 siRNA may be able to inhibit invasion and metastasis of tumor cells rather than tumor growth.

It has been reported that inhibition of PLK1 expression suppressed invasion of tumor cells and decreased the activity of cluster of differentiation (CD)44v6 (23), which is an important member of the cell adhesion molecule CD44 family. Additionally, HSF1 serves various important roles in cancer via regulating cell proliferation, anti-apoptosis, epithelial-mesenchymal transition, migration, invasion and metastasis (24). HSF1 may promote invasion and metastasis of hepatocellular carcinoma by enhancing cell motility through Hsp27 (25). Such findings indicated that PLK1 and HSF1 may be involved in the regulation of cancer metastasis.

Intravenous injection of PLK1 siRNA with a fusion protein of human epidermal growth factor 2-single-chain fragmented antibody and protamine peptide retarded breast tumor growth and reduced metastasis (12). Furthermore, it has been reported that melanoma cells infected with adenovirus expressing HSF1 shRNA markedly reduced invasion and metastasis in a subcutaneous xenograft model (11). In the present study, injections of HSF1 siRNA or PLK1 siRNA with cationic liposomes inhibited tumor progression in HeLa metastasis. These findings suggest that PLK1 and HSF1 are critical factors that influence metastasis and have an important role in tumor progression.

In conclusion, injection of PKL1 siRNA or HSF1 siRNA into mice with liver HeLa metastasis inhibited tumor metastasis. PLK1 and HSF1 may, therefore, be considered as promising therapeutic targets for tumor metastasis.

\section{Acknowledgements}

The present project was supported in part by a Grant-in-Aid for Scientific Research (C) from the Japan Society for the Promotion of Science (KAKENHI; grant nos. JP26460046 and JP17K08251).

\section{References}

1. Sorger PK: Heat shock factor and the heat shock response. Cell 65: 363-366, 1991.

2. Morano KA and Thiele DJ: Heat shock factor function and regulation in response to cellular stress, growth, and differentiation signals. Gene Expr 7: 271-282, 1999. 
3. Wang Y, Theriault JR, He H, Gong J and Calderwood SK Expression of a dominant negative heat shock factor-1 construct inhibits aneuploidy in prostate carcinoma cells. J Biol Chem 279: 32651-32659, 2004

4. Calderwood SK: Heat shock proteins in breast cancer progression--a suitable case for treatment? Int J Hyperthermia 26: 681-685, 2010.

5. Schmucker S and Sumara I: Molecular dynamics of PLK1 during mitosis. Mol Cell Oncol 1: e954507, 2014.

6. Kim SA, Yoon JH, Lee SH and Ahn SG: Polo-like kinase 1 phosphorylates heat shock transcription factor 1 and mediates its nuclear translocation during heat stress. J Biol Chem 280 12653-12657, 2005.

7. Takai N, Hamanaka R, Yoshimatsu J and Miyakawa I: Polo-like kinases (Plks) and cancer. Oncogene 24: 287-291, 2005.

8. Knecht R, Elez R, Oechler M, Solbach C, von Ilberg C and Strebhardt K: Prognostic significance of polo-like kinase (PLK) expression in squamous cell carcinomas of the head and neck. Cancer Res 59: 2794-2797, 1999.

9. Takahashi T, Sano B, Nagata T, Kato H, Sugiyama Y, Kunieda K, Kimura M, Okano Y and Saji S: Polo-like kinase 1 (PLK1) is overexpressed in primary colorectal cancers. Cancer Sci 94 $148-152,2003$.

10. Kim SA, Kwon SM, Yoon JH and Ahn SG: The antitumor effect of PLK1 and HSF1 double knockdown on human oral carcinoma cells. Int J Oncol 36: 867-872, 2010.

11. Nakamura Y, Fujimoto M, Fukushima S, Nakamura A, Hayashida N, Takii R, Takaki E, Nakai A and Muto M: Heat shock factor 1 is required for migration and invasion of human melanoma in vitro and in vivo. Cancer Lett 354: 329-335, 2014.

12. Yao YD, Sun TM, Huang SY, Dou S, Lin L, Chen JN Ruan JB, Mao CQ, Yu FY, Zeng MS, et al: Targeted delivery of PLK1-siRNA by $\mathrm{ScFv}$ suppresses Her2+ breast cancer growth and metastasis. Sci Transl Med 4: 130ra48, 2012.

13. Zhang S, Zhi D and Huang L: Lipid-based vectors for siRNA delivery. J Drug Target 20: 724-735, 2012.

14. Simberg D, Weisman S, Talmon Y, Faerman A, Shoshani T and Barenholz Y: The role of organ vascularization and lipoplex-serum initial contact in intravenous murine lipofection. J Biol Chem 278: 39858-39865, 2003
15. Hattori Y, Arai S, Kikuchi T, Ozaki K, Kawano K and Yonemochi E: Therapeutic effect for liver-metastasized tumor by sequential intravenous injection of anionic polymer and cationic lipoplex of siRNA. J Drug Target 24: 309-317, 2016.

16. Hattori Y, Arai S, Okamoto R, Hamada M, Kawano K and Yonemochi E: Sequential intravenous injection of anionic polymer and cationic lipoplex of siRNA could effectively deliver siRNA to the liver. Int J Pharm 476: 289-298, 2014

17. Lee YJ, Kim EH, Lee JS, Jeoung D, Bae S, Kwon SH and Lee YS: HSF1 as a mitotic regulator: Phosphorylation of HSF1 by Plk1 is essential for mitotic progression. Cancer Res 68: 7550-7560, 2008.

18. Seth S, Matsui Y, Fosnaugh K, Liu Y, Vaish N, Adami R, Harvie P, Johns R, Severson G, Brown T, et al: RNAi-based therapeutics targeting survivin and PLK1 for treatment of bladder cancer. Mol Ther 19: 928-935, 2011

19. Jacobs AT and Marnett LJ: HSF1-mediated BAG3 expression attenuates apoptosis in 4-hydroxynonenal-treated colon cancer cells via stabilization of anti-apoptotic $\mathrm{Bcl}-2$ proteins. J Biol Chem 284: 9176-9183, 2009.

20. Livak KJ and Schmittgen TD: Analysis of relative gene expression data using real-time quantitative PCR and the 2(-Delta Delta C(T)) method. Methods 25: 402-408, 2001

21. Sakurai Y, Hatakeyama H, Akita $\mathrm{H}$ and Harashima $\mathrm{H}$ : Improvement of doxorubicin efficacy using liposomal anti-polo-like kinase 1 siRNA in human renal cell carcinomas. Mol Pharm 11: 2713-2719, 2014.

22. Kato M, Hattori Y, Kubo M and Maitani Y: Collagenase-1 injection improved tumor distribution and gene expression of cationic lipoplex. Int J Pharm 423: 428-434, 2012.

23. Zhang XG, Lu XF, Jiao XM, Chen B and Wu JX: PLK1 gene suppresses cell invasion of undifferentiated thyroid carcinoma through the inhibition of CD44v6, MMP-2 and MMP-9. Exp Ther Med 4: 1005-1009, 2012.

24. Jiang S, Tu K, Fu Q, Schmitt DC, Zhou L, Lu N and Zhao Y: Multifaceted roles of HSF1 in cancer. Tumour Biol 36: 4923-4931, 2015.

25. Fang F, Chang R and Yang L: Heat shock factor 1 promotes invasion and metastasis of hepatocellular carcinoma in vitro and in vivo. Cancer 118: 1782-1794, 2012. 